\section{JAMIESON'S FIRST NOTEBOOK OF HEAD INJURY. Third} Edition. By Brian North. Published by Butterworths. 116 pages. $\$ 12 \mathrm{Cdn}$. approx.

This short and entertaining volume dedicated to the basics of head injury is a 1983 update of the original volume written by Dr. Kenneth G. Jamieson of the University of Queensland (Australia) in 1984 and subsequently updated in 1971.

Dr. Jamieson's original aim was to produce a short and simple volume directed primarily to medical students and rural practitioners and nurses in the Australian environment to assist them in their care of the head injured when travel distances to a neurosurgical centre are great.

Resultant upon Dr. Jamieson's untimely death in 1976, Dr. Brian North (Director of Neurosurgery, Royal Adelaide Hospital, Adelaide, South Australia) has undertaken the current update.

This volume carries out very well its primary purpose of remaining simple and straight forward and being directed to those who know nothing or little about the care of head injuries.

There is no question that the book is directed to the "neurosurgically uninitiated" and this volume should be considered in no way a reference for neurologic or neurosurgical trainees.

I would consider this book most valuable in the libraries of medical students, clinical clerks, surgical residents undertaking a transient neurosurgical rotation, rural physicians and rural hospital emergency departments. The entertaining, and often humorous style of writing, use of rhyme and use of diagrams serve to emphasize important points. For example, in the section related to chronic subdural hematoma, the following is found -

An old man was found on the ground -

Some days he'd almost come round.

They stood and debated

While he fluctuated,

At P.M. a large clot was found.

In light of this volume's brevity, clarity, easy readibility and inexpensiveness it is recommended to the categories of readers noted above.

K.M. Hunter, Calgary, Alberta

THE NEUROMUSCULAR JUNCTION. 1984. Edited by Brumback and Gerst. Published by Futura Publishing Company, Mount Kisco, New York. 354 pages.

It is a surprise that 354 pages are now required to detail the complexities of the structure, function, and pathology of the neuromuscular junction. In this volume, the multiple authors provide current and detailed accounts of the light and electronmicroscopic features of the end plate, of the process of neuromuscular transmission and of the actions of cholinesterases, depolarizing and non depolarizing drugs and anticholinesterases at the end plate. A chapter on the effects of nerve injury primarily addresses the issue of muscle function in denervation and its relevance in this volume is questionable. The final two chapters deal with the clinical disorders of the neuromuscular junction. Donald Sanders covers myasthenia gravis and its pathogenesis, clinical diagnostic features and management fully and accurately. The chapter dealing with other neuromuscular disorders is less successful. After descriptions of miscellaneous neuromusclar disorders including the myasthenic syndrome, congenital myasthenia and botulism, a good deal of information is offered on drugs, toxins and venoms that remains to be better-defined in the light of our newer knowledge and ability to understand altered neuromuscular function.

This book is a comprehensive, very detailed and thorough examination of the mammalian neuromuscular junction. It will be used mainly as a reference source for a basic understanding of the structure and function of the end plate. It will appeal particularly to pharmacologists and physiologists, but should be familiar territory for anaethetists and clinicians dealing with neuromuscular disorders.

John G. Humphrey, Toronto, Ontario

MUSCLE PATHOLOGY. 1984. Edited by Reid R. Heffner, Jr. Churchill-Livingstone. New York, Edinburgh, Melbourne. 235 pages, $160 \mathrm{~B} \& \mathrm{~W}$ illustrations. $\$ 56 \mathrm{Cdn}$. approx.

This short monograph on muscle pathology is one of the Churchill-Livingstone series 'Contemporary Issues in Surgical Pathology'. The twelve chapters were written by 14 contributing authors, all from the United States. The book joins several other recent texts specifically addressing the needs of general pathologists who interpret muscle biopsies but do not require long, supplementary discussions of clinical, electromyographic, and biochemical aspects. As such, the chapters are not organized according to clinical classifications of disease, but rather on specific categories of pathologic changes seen histologically and special techniques. The importance of accurate diagnosis of histochemistry, immunofluorescence, and electron microscopy are appropriately emphasized in most chapters.

The first chapter is supposed to provide a 'clinical approach to neuromuscular disease', but is so general that it is suited only for a textbook for medical students. The table of classical classifications of diseases regrettably still lists oculopharyngeal myopathy as a muscular dystrophy, ignoring the numerous publications of the last decade demonstrating mitochondrial abnormalities as the basis of this disease; this error is rectified by Bauserman and Heffner in a later chapter (8) devoted exclusively to mitochondrial myopathies. Chapter 2 deals with the collection and preparation of the biopsy specimen. It contains no information on the technical problems of histochemical preparation. The recommended cryostat sections of $10 \mu \mathrm{m}$ are thicker than the standard $6 \mu \mathrm{m}$ used in most laboratories and could result in loss of cytologic detail, particularly in infant muscle, and too dark histochemical staining with loss of contrast between fiber types. Chapter 3 on histochemistry contains an erroneous assertion that type II fiber atrophy is characteristic of early denervation, which could lead to serious errors in diagnosis by inexperienced pathologists.

Chapter 3 by Cancilla on general reactions of muscle to injury is concise, well organized, clearly written, and contains carefully selected photo-micrographs. Another chapter (5) by Bennington and Krupp, entitled 'Morphometric analysis of muscle', is an excellent, erudite summary of a complex topic and alone is worth the price of the book. 
Fluorescence microscopy of muscle is the subject of Chapter 6 by Little and Perl. This is another concise, informative, and interesting discussion by authors who have made major contributions in the application of fluorochromic stains of nucleic acids for the demonstration of denervated and regenerating myofibers. I would have enjoyed elaboration of their brief comments on the potential uses for infrared microscopy. Regrettably the publisher did not see fit to include even one color illustration in this chapter. Unlike histochemical stains such as ATPase in which contrasts of staining intensity are convincingly illustrated in black-and-white, fluorochromic stains such as acridine orange rely on differences of wavelength of emitted light perceived as different colors, and the striking if not beautiful pathologic findings cannot be captured on blackand-white film. The publisher's business manager won this skirmish over the scientists!

An interesting chapter (7) entitled 'Core-genic myopathies' was contributed by S.M. Chou, who has done much experimental work in elucidating the pathogenesis of central cores and target fibers. Chapter 9 on glycogenoses and lipidoses of muscle is well written by Schochet, another recognized authority with a background of many original contributions.

Chapters 10 by Dudley and 11 by Heffner on generalized and focal inflammatory myopathies respectively describe the principal pathologic findings in this important category, including such associated changes as focal denervation, altered glycogen and lipid metabolism, sarcotubular profiles, and changes in endothelial cells. Myositis ossificans and fasciitis receive more thorough discussions than usually found in books on muscle pathology. The final chapter (12) by Sharon Weiss on neoplasms of muscle is thorough in its scope and its classical histologic descriptions, but does not address the newer histochemical techniques as a supplement to the diagnosis of such tumors as rhabdomyosarcoma. Only peroxidase-antiperoxidase for the localization of myosin and myoglobin in tumor cells is mentioned in one sentence.

I would conclude that this book is well worth the purchase price and contains much useful and practical information for the anatomical pathologist, but it is not a comprehensive text. Particular deficiencies include the lack of a chapter on congenital myopathies and the special problems of infant biopsies, absence of color illustrations, lack of discussion of technical artifacts that could be misinterpreted as pathologic changes, and no practical methods are offered for preparing common histochemical stains, for inexperienced pathologists wishing to establish a muscle biopsy service in their laboratories. (Originally published in Laboratory Medicine. Reproduced with permission of the American Society of Clinical Pathologists).

Harvey B. Sarnat, Calgary, Canada

EXPERIMENTAL ALLERGIC ENCEPHALOMYELITIS: A USEFUL MODEL FOR MULTIPLE SCLEROSIS. 1983. Edited by E.C. Alvord, Jr., M.W. Kies and A.J. Suckling. Published by Alan R. Liss, Inc. Company, New York. 554 pages.

The editors have assembled the papers which were presented at a satellite conference of the International Society of Neurochemists which was held at the University of Washington, Seattle, July 16, 1983. The central theme of the book is the usefulness of chronic relapsing EAE as a model for the study of Multiple Sclerosis.

The book is well organized in major sections, each of which focuses on a major subject relevant to EAE. In each chapter there are 5 to 8 contributions, largely derived from field leaders. Only occasionally does the organization break down. In Chapter 7 there are several contributions which would have better complimented Chapters 3 or 4 .

The number of contributors exceeds 100 and as might be expected there are some strong contributions and some particularly weak contributions. In any case the collection of papers allows a reader an impression of the state of the art of research in this area. Each of the individual contributions is brief and easy to read. Much of the material has been published elsewhere. Each major subdivision in this 554 page tome was introduced by a succinct comment by a major investigator, and the papers were all summarized appropriately in a concluding note.

This book will be a valuable addition to any neuroscience or neuroimmunology library, and to any neurologist who has followed the developments in this field over the last 25 years.

G.P.A. Rice,

London, Ontario 